\title{
Influence of within pen gender composition and weight variation on the welfare and growth performance of finishing pigs
}

S Conte ${ }^{1,2}$, L Boyle $^{2}$, P Lawlor ${ }^{2}, \mathrm{~N} \mathrm{O}^{\prime}$ Connell ${ }^{1}$

${ }^{1}$ Queen's university, Belfast, United Kingdom, ${ }^{2}$ Teagasc, Cork, Ireland

Email: sabine.conte@teagasc.ie

Introduction Research shows that entire male pigs are more aggressive during the finishing period than female pigs (Boyle and Björklund 2007). It is not clear to what extent this aggression is influenced by the gender composition of the group. The level of within-group weight variation is also likely to influence aggression and productivity in pigs (O'Connell et al. 2005). The aim of this study was to assess the effects of housing entire male finishing pigs in single-sex or mixed-sex groups with high or low within-group weight variation. Effects on aggressive and sexual behaviour and on injury levels and productivity were assessed.

Material and methods At the start of the finishing period, 200 pigs were allocated to one of 4 treatments (over 5 replicates) according to group gender composition (all-male group of 10 pigs, or mixed-gender group (5 males, 5 females)) and within-group weight variation (high variation, $c v \sim 0.18$ or low variation, $c v \sim 0.05$ ). Treatments were: all-male with high variation $[\mathrm{MaH}]$, all-male with low variation $[\mathrm{MaL}]$, mixed-sex with high variation $[\mathrm{MiH}]$ and mixed-sex with low variation [MiL]. The initial mean body weight in the pen was balanced across treatments $(29 \pm 0.3 \mathrm{~kg})$. Pigs were provided with ad-libitum access to food. The frequency of fighting and mounting was recorded during 12 focal 3-min observations each week across the 11 week finishing period. Skin lesions were scored at 5, 35 and 70 days after mixing, using a scale of 0 to 4 (0: no lesion, 1: one superficial lesion, 2: more than one superficial or one red lesion, 3: more than one red lesion, 4: one red open wound) on 16 areas of the body of each pig. Scores for each area of the body were combined. Pigs were weighed at 10 and 21 weeks of age, and daily feed intake (DFI) was recorded. The DFI, the average daily gain (ADG) and the food conversion ratio (FCR) were calculated. Pigs were slaughtered at 21 weeks of age and data on carcass weight, $\mathrm{P}_{2}$ fat depth, lean meat and kill out $\%$ were collected. The within-group variation in slaughter weight, carcass weight, $\mathrm{P}_{2}$ fat depth and lean meat \% was calculated. For all data, the pen was considered as the experimental unit. Skin lesion scores and frequency of fighting and mounting were analysed by REML variance components analysis with day, gender composition and within-group weight variation as main effects. Data on performance were analysed by ANOVA with gender composition and within-group weight variation as main effects. For the analysis of growth performance, initial mean body weight was used as a covariate.

Results The average frequency (per 3-min observation) of mounting was 2-fold higher in all-male than in mixed-sex groups (all-male group: 0.22, mixed-sex group: 0.11; s.e. 0.031; $\mathrm{P}<0.001$ ). There was no effect of within-group weight variation on the frequency of mounting $(\mathrm{P}>0.05)$, and no effect of treatments on the frequency of fighting $(\mathrm{P}>0.05)$. Skin lesion scores were highest in MaL groups and lowest in MiH group (MaL: 1.25, MaH: 1.15, MiL: 1.12, MiH: 1.09; s.e. 0.056, $\mathrm{P}<0.05)$. There was a tendency for interactive effects between group gender composition and within-group weight variation on slaughter weight, carcass weight, DFI and ADG $(\mathrm{P}<0.10)$, but no effect on the FCR $(\mathrm{P}>0.05$, Table 1$)$. Reducing within-group starting weight variation led to a reduction in within-group variation in weight at slaughter (high variation: 0.15 , low variation: 0.08 ; s.e $0.010, \mathrm{P}<0.001$ ) and carcass weight (high variation: 0.16 , low variation: 0.09 ; s.e. $0.010, \mathrm{P}<0.001)$. The $\mathrm{P}_{2}$ fat depth and lean meat $\%$ were not affected by treatment $(\mathrm{P}>0.05$, Table 1$)$.

Table 1 Interactive effects between group gender composition and within-group weight variation on production performance

\begin{tabular}{lllllll}
\hline \hline & $\mathrm{MaH}$ & $\mathrm{MaL}$ & $\mathrm{MiH}$ & $\mathrm{MiL}$ & $\mathrm{s.e}$ & $\mathrm{P}$ \\
\hline Slaughter weight, kg & 87.1 & 89.0 & 88.5 & 86.0 & 1.27 & $<0.1$ \\
Carcass weight, kg & 64.5 & 66.4 & 66.9 & 64.5 & 1.10 & $<0.1$ \\
DFI, g & 1757 & 1888 & 1867 & 1795 & 55.8 & $<0.1$ \\
ADG, g & 705 & 780 & 746 & 735 & 23.6 & $<0.1$ \\
FCR & 2.50 & 2.42 & 2.51 & 2.45 & 0.040 & $\mathrm{NS}$ \\
P $_{2}$ fat depth, mm & 11.1 & 12.0 & 11.3 & 11.1 & 0.42 & $\mathrm{NS}$ \\
Lean meat, \% & 62.1 & 61.3 & 61.9 & 62.1 & 0.34 & $\mathrm{NS}$ \\
\hline \hline
\end{tabular}

Conclusions Housing finishing entire male pigs in all-male groups led to an increase in injuries when initial within-group weight variation was low, and led to increased levels of mounting, which is likely to have a negative effect on welfare. This suggests that entire male pigs should be housed in mixed-sex groups. Reducing the within-group weight variation at the start of the finishing period led to reduced within-group variation in slaughter and carcass weight. This is beneficial in terms of leading to a more efficient use of finishing accommodation, and reducing variability in the final product. However, in mixed-sex groups this practice led to slight numerical reductions in performance parameters, and increases in injury scores.

Acknowledgements The authors gratefully acknowledge funding from Teagasc Walsh fellowship.

\section{References}

Boyle, L. and Björklund, L. 2007. Animal Welfare 16, 259-262.

O’Connell, N.E., Beattie, V.E. and Watt, D. 2005. Livestock Production Science 97, 107-115. 